\title{
Costs of Routine Immunization Services in Moldova: Findings of a Facility-Based Costing Study
}

\section{Introduction}

Goguadze K. ${ }^{1}$, Chikovani I. ${ }^{1}$, Gaberi C. ${ }^{2}$, Maceira D. ${ }^{3}$, Uchaneishvili M. ${ }^{1}$, Chkhaidze N.1 Gotsadze G1 .

${ }^{1}$ Curatio International Foundation (CIF); 37d Chavchavadze ave. 0162, Tbilisi, Georgia

${ }^{2}$ Ministry of Health of the Republic of Moldova; 67A Gh. Asachi str. Chișinău

${ }^{3}$ Center for the Study of State and Society (CEDES); Buenos Aires, Argentina 


\section{Abstract}

Objective: Available estimates on how much it costs to provide routine immunization services are out-dated. This study attempts to address gaps in evidence by evaluating the total economic and unit costs of delivering routine immunization (RI) services in Moldova as part of a multi-country study on the costs and financing of routine immunization (EPIC).

Methods: This cross-sectional study is based on a multistage stratified random sample of fifty primary health care facilities. Data on inputs, prices, and outputs were collected retrospectively for 2011 and analysed using an ingredient-based costing approach in Excel and SPSS ${ }^{\text {. }}$

Results: The average total annual facility cost for RI was $\$ 11,943$ ranging from $\$ 565$ to $\$ 112,548$ and labour cost was the main driver of routine immunization costs contributing $65 \%$, followed by capital costs $-16.3 \%$ and the cost of vaccines and injection supplies accounting for $9 \%$.

The average cost per dose was $\$ 18.3$, the cost per child was $\$ 316.6$ and the cost per fully immunized child was $\$ 332.3$. The results show considerable variation in the costs of routine immunization services across facility type and depending on a facility scale i.e. annual doses administered.

Conclusions: The study shows that the cost of fully immunizing a child in a middleincome country is much higher than previous estimates. These results will be used by the government for better planning and financing of routine immunization services, leading to greater sustainability. 


\section{Introduction}

Vaccination against childhood communicable diseases through the Expanded Program on Immunization (EPI) is one of the most cost-effective public health interventions [1]. By reducing mortality and morbidity, vaccination can substantially contribute toward achieving the Millennium Development Goal of reducing under-five mortality among children by two-thirds between 1990 and 2015 [2].

The costs and financing of national immunization programs have been evaluated since the 1980s as part of the Universal Childhood Immunization Initiative [3]. However, currently available information is out-dated and new knowledge on the full economic costs of routine immunization programs is important to inform both global and national policies and practice.

Our study evaluates the total economic and unit costs of the immunization program in the Republic of Moldova as part of a multi-country study supported by the Bill and Melinda Gates Foundation and aimed at evaluating the costs of routine immunization programs and new vaccine introduction. It is expected that our study results will contribute to building a new evidence-base that will provide valuable inputs into the development of national and global policies, as well as contribute to better planning and management of the national immunization program in Moldova.

\section{The National Immunization Program in Moldova}

The current plan for the national immunization program in Moldova covers the period 2011-2015 and guarantees free immunization against ten infectious diseases: poliomyelitis, diphtheria, tetanus, pertussis, hepatitis B, measles, mumps, rubella, tuberculosis, and Haemophilus influenza type B. Based on this program Moldova plans to increase DTP 3 coverage up to 94\% and 95\% in 2014 and 2015 
respectively, although country already reports high DPT3 coverage rate that is above $90 \%[4,5]$.

Routine immunization services ${ }^{1}$ are delivered primarily through primary health care (PHC) facilities. In urban areas, Family Medicine Centres (FMC) serve a population of between 40,000 to 80,000 and 440-880 infants. In rural areas, Health Centers (HC) cater to 4,500 inhabitants and around 50 infants; Family Doctor Offices (OFD) serves between 900-3,000 inhabitants and about 10-33 infants; and, small Health Offices (HO) cover up to 900 residents and 5-8 infants. All facilities with the exception of HOs are staffed with doctors and nurses, while most of HOs only employ nurses. Private facilities, though growing in number, do not engage in the delivery of the routine immunization services funded by the state. In all PHC facilities, immunization is delivered as a fixed strategy and no outreach activities are carried out. Consequently, costs captured in this study only pertain to the fixed strategy.

\section{Material and Methods}

This is a cross-sectional facility-based costing study, which examines total and unit costs of routine immunization and describes cost drivers and cost variation across facilities in Moldova.

\section{Facility Sampling}

The study was based on a representative sample of PHCs selected based on multistage, stratified random sampling. In the first stage, the total number of vaccine doses delivered in 2011 was used to stratify 37 districts into three strata:

\footnotetext{
1 Routine immunization services are defined as those immunization services or activities that are conducted regularly as part of the national program.
} 
districts with low, medium and high vaccine doses administered per annum. Stratification was based on tercile groups derived through simple frequency analysis on annual doses administered. After stratification two districts were chosen in each stratum using simple random sampling approach, resulting in 6 districts in the sample.

In the second stage, PHC facilities were randomly selected from a complete list of facilities stratified by urban and rural location within each sampled district. Proportions of urban/peri-urban and rural facilities from the total number of facilities were estimated, and used to calculate the number of rural and urban/ periurban facilities to be sampled. One peri-urban facility was randomly selected in each sampled district and three urban facilities were randomly selected from the capital city. Rural facilities were selected using systematic random sampling. Consequently, the final sample consisted of eight urban/peri-urban and 42 rural facilities, represented by five FMCs, 10 HCs, 23 OFDs and 12 HOs. This sample was drawn from 1,318 facilities involved in the delivery of immunization services.

Data collection in facilities took place from October 3rd 2012 to January $14^{\text {th }} 2013$ using standardized, pre-tested and structured questionnaires. Data were collected by experienced data collectors and included interviews with facility administrators and health workers involved in immunization, facility observation, and record review.

\section{Cost analysis}

The data for the routine EPI were collected retrospectively for 2011 calendar year and converted into \$US 2011 using the current exchange rate for the year. Cost calculations were based on common approach methodology for the costing and financing analysis of routine immunization program [6,7] and costing guideline for 
comprehensive Multi-Year Plan (cMYP) [8]. Costs of all inputs were captured using an ingredients approach listing all inputs by activity and quantities and prices for each input element [9] [10].

The cost data included a comprehensive list of capital ${ }^{2}$ as well as recurrent expenditure items. Capital costs were analyzed using a 3\% discount rate [11] and country specific useful life years for different capital items were applied [12].

The cost of labor included salaries and other allowances for the staff involved in the EPI and were calculated based on a self-reported percentage of time spent by staff on different immunization activities.

The cost of vaccines and syringes were calculated for each vaccine separately. The number of doses used included both doses administered (for all vaccines in the schedule) and doses wasted, which allowed estimating vaccine-specific and overall facility level wastage rates [Error! Bookmark not defined.].

Cold chain costs were calculated by inventorying the number and type of cold chain equipment and the World Health Organization (WHO) product information sheets [13] informed the prices. Cold chain energy costs were based on energy consumption characteristics from the WHO Products Information Sheets and local unit price of $0.095 \$ \mathrm{US} / \mathrm{kw} / \mathrm{h}$ was applied.

For those costs not exclusive to immunization, the following allocation methods were used: cost of vehicles and vehicle maintenance were allocated based on the proportion of $\mathrm{km}$ travelled for routine immunization out of total $\mathrm{km}$ travelled in 2011; building costs were allocated based on the proportion of square meters designated for routine immunization (where vaccines are administered, stored) out of total facility space. More details about the costing, unit prices are available from

\footnotetext{
${ }^{2}$ Capital items include buildings, vehicles, cold chain equipment and office equipment
} 
elsewhere [Error! Bookmark not defined.] A table of key assumtions/cost allocation methods is provided in the Annex.

Estimated costs were converted into the cost per dose delivered, cost per Fully Immunized Child (FIC) and cost per infant. For the purposes of our study the Fully Immunized Child denotes a child less than one year of age who received three doses of DTP vaccine (DTP3). Although this definition differs from what is being formally used in Moldova ${ }^{3}$, it is useful for international comparability of costs.

Excel software was used for calculation of various cost elements and unit costs for a facility. These calculated variables were transferred to SPSS version 19.0 for further statistical analysis.

Throughout the paper, unless otherwise noted, we present total economic costs of the routine immunization services on a facility level in 2011 \$US at the current exchange rate. The costs and cost variations are presented by urban-rural location, type and scale of a facility using T-test for statistical significance.

\section{Results}

\section{Total facility costs and observed cost variation}

The total facility level immunization costs in the sample varied from $\$ 565$ to $\$ 112,548$, with a mean of $\$ 11,943$ and median $\$ 3,822$ \$US. Log transformed variables reveal a positive linear correlation between total facility level costs and total doses delivered $(\mathrm{r}=0.95, \mathrm{p} \varangle 0.01)$.

Figure 1 shows that total facility cost varies by facility type, with costs increasing with facility size (HOs to FMCs). Variation within facility type is not large with the

\footnotetext{
3 FIC in Moldova denotes a child less than one year of age who received three doses of DTP-Hib-Hep B, one doses of BCG, one doses of Hep B, three doses of OPV and one doses of MMR vaccines.
} 
exception of FMCs where costs range from $\$ 28,335$ to $\$ 112,548$ (mean $\$ 62,902$ ).

Two facilities within each of the HOs and HCs have significantly higher costs compared to their peers. Both of these facilities were found to be high performing ones delivering more doses annually and achieving higher coverage rates. The figure also shows that while the majority of facilities achieve high DTP3 coverage rates, a few underperforming facilities contribute to a lower average DTP3 coverage rate in the sample - 94.8\%. HCs and OFDs had higher coverage rates (98.1-97.8\%, respectively), while HOs have the lowest rates on average (90.2\%). The study also found that the facility total cost correlates with the number of infants covered by the facility $(\mathrm{r}=0.95, \mathrm{p} \varangle 0.01)$.

\section{Total Facility Cost Structure}

Analysis revealed that the share of labor costs increases with facility size and scale (defined as total doses delivered in 2011), and are the main driver of immunization costs. Labor cost accounted for $65 \%$ of the total facility immunization cost for the sample, ranging from $55 \%$ in lower scale to $73 \%$ in higher scale facilities. Close to $87 \%$ of labor inputs are spent on four critical immunization functions/ activities: routine service delivery $(29.8 \%$ of the time), program management $(23.5 \%)$, recordkeeping and health management information (17.1\%), and social mobilization (16.7\%) (see Figure A1 supplied in annex for more details).

Capital costs are the second biggest contributor to total facility immunization costs. The share of recurrent and capital costs differed across facilities as well. Namely, the share of capital costs was lowest in FMCs - 10.3\% and highest in HOs - 22.8\%, with the average across all sampled facilities being 16.3\% (see Table 1). Furthermore, in the facilities with higher scale, the share of capital costs was around $9.3 \%$, while in a low scale facility it reached $23.1 \%$. Vaccine and injection supplies only accounted for 
$8.72 \%$ of the cost (range: $8.3 \%-14.42 \%$ by facility type and $8.04 \%-10.63 \%$ by facility scale).

\section{Facility Unit costs and their variation}

A range of unit costs was evaluated for this study, including cost per dose, cost per infant, and cost per FIC and amounted to 6.4 \$US, 112.3 \$US and 117.8 \$US respectively. Differences between urban and rural facilities are presented in Table 2. These differences (both with and without shared labor costs) are not statistically significant. Also differences in the unit costs captured between types of facilities did not reveal statistical significance. However, when shared labor costs were removed, the cost per dose was $\$ 3.10$ in the sample of FMCs, increasing to $\$ 8.50$ per dose in the sample of HOs, which became statistically significant ( $p \varangle 0.01$ ).

Figure 2 shows a negative relationship between facility scale and unit cost for the facilities in the sample: larger facilities have lower unit costs in general.

The only significant influence, although with marginal statistical significance $(\mathrm{P}<0.1)$ was between unit costs and facility scale and only for the cost per FIC and/ or cost per infant. The statistical significance of scale influence increased when shared labor costs were removed from unit cost estimates (see Table 2).

\section{Unit Costs and Facility Level Immunization Program Performance}

Figure 3 shows the relationship of facility unit costs and performance measured as DPT3 coverage rate 4 . HOs with the lowest DPT3 coverage rates spend a comparable amount per dose delivered as OFDs and HCs. The amounts of non-labor inputs are highest in HOs and lowest in FMCs and largely determined by variable use of capital.

\footnotetext{
4 To calculate DPT3 coverage rate we used number of children receiving three doses of DTP (or DTP containing vaccine) under the age one divided by the number of children under one in the facility catchment area, determined through facility records.
} 
HCs spend the highest amount per dose delivered, but also achieve the highest coverage rates. FMCs seems to be the most efficient facilities being able to deliver immunization at the lowest cost per dose by using labor and non-labor inputs effectively. Finally, while HCs spend the highest amount per dose they spend the least (after FMCs) on non-labor inputs.

\section{Discussion}

The study helped estimate average unit cost per dose delivered in 2011 (\$18.3) and cost per FIC (\$316.6). These figures are higher than what has been previously reported in the literature [Error! Bookmark not defined.] [14] [15] [16] and also higher than the figures used in the CMYP - 191 \$US per FIC [Error! Bookmark not defined.]. Though cMYP underestimated shared costs by $32 \%$ and capital costs by $61 \%$, but recurrent costs were overestimated by almost 44\% [Error! Bookmark not defined.].

Our study shows that the labor inputs are significant contributor to a unit cost and consequently to the overall immunization program cost. These findings are comparable with the evidence documented elsewhere i.e. immunization program being labor intensive [Error! Bookmark not defined.] [Error! Bookmark not defined.] [Error! Bookmark not defined.]. Furthermore, the study captured the breakdown of labor inputs on various functions, not easily available from other studies, showing that four activities on a facility level determine close to $87 \%$ cost of labor inputs for immunization. The government of Moldova is focused on increasing health system efficiency. These findings suggest that reducing staff time spent on immunization could help increase efficiency of the program and it could be achieved 
either through task shifting $[17]^{5}$ i.e. delegating certain immunization related tasks from doctors to nurses, or through reducing time spend on management and/or recordkeeping functions. The latter could be achieved with the help of computer based HMIS, which is currently being developed. Adequate modules for immunization program planning and management along with the modules for record keeping offer potential for reducing staff time spent on these functions, conditioned that PHC modules of the HMIS are developed with this objective in mind.

We have also seen variable use of capital by facility type and by scale. Namely, larger facilities (i.e. FMCs and HCs) and facilities with a greater scale are able to use capital more effectively. The influence of the capital becomes significant when shared labor costs are excluded and when variable use of capital becomes critical determinant of the costs. The level of scale is associated with lower unit costs, and supports the available global evidence [Error! Bookmark not defined.] [18]. Consequently increasing the scale of a facility (by merging smaller ones into larger ones, where possible) could help reduce the program unit costs. Although this may as well increase distance to a facility and may negatively affect immunization coverage rates.

Furthermore, vaccines and vaccine supplies were found to be the third most important cost driver of the immunization costs on a facility level. Consequently reducing vaccine prices, which currently are double the prices of UNICEF supplied

\footnotetext{
${ }^{5}$ Task shifting is the name given to a process of delegation whereby tasks are moved, where appropriate, to less specialized health workers. By reorganizing the workforce in this way, task shifting presents a viable solution for improving health care coverage by making more efficient use of the human resources already available.
} 
vaccines [19], could be the strategy for the country to consider. Furthermore, Moldova is graduating from the GAVI Alliance in 2016 and country is contemplating reforming its immunization program and decentralizing vaccine procurement responsibilities due to the nature of the national public finance regulations. Our study shows that the cost of vaccines is critical element of the immunization program and needs to be well managed. Therefore centralized model for vaccine procurement could be more effective, while decentralization of this function may drive vaccine prices up and could increase overall program cost.

Finally, we have seen that Moldova achieves high immunization coverage rates compared to other countries in the region [20]. However, differences exist when coverage rates are examined by facility level. Namely, HOs that are equipped only with nurses and located in small villages have the lowest DPT3 coverage. However, due to a very small number of infants - 7 (95\%CI: 6.7-7.9) in the facility catchment area this low coverage translates into at most one child missing its third dose of DPT. HCs along with OFDs achieve higher DTP3 coverage rates compared with others. It could be assumed that relatively small size of the catchment population and having doctors on staff probably allows these facilities to better identify, plan and follow-up infants and consequently achieve higher DPT3 coverage. While FMCs also have doctors on staff, due to the large size of the catchment population, they may face additional complexities in finding, following and immunizing a child. In light of this increasing DPT3 coverage could be achieved by placing more importance on FMCs, where immunization program performance is the second poorest after HOs - 92.7\%. Improving performance of these facilities seems more feasible on several counts: they have better staffing and more human resources, they have more infants and improving their performance would have greater impact on the national coverage rate. Also, due to their ability to deliver most cost-efficient 
immunization services it may be less costly option for the national budget. However, this strategy raises urban-rural equity concerns. To mitigate these concerns, it seems necessary to focus managerial efforts on significantly underperforming rural facilities, where coverage rates are below $80 \%$ and provide supportive supervision or other assistance that will be necessary.

While the study provides valuable evidence, its strength and limitations should be kept in mind. Namely, this is the first facility-based costing study of immunization services conducted in Moldova and provides rich data that could be used in the national planning as well as for benchmarking results of future research. The sample size at 50 facilities was relatively small and excluded maternity homes, where BCG and Hepatitis B birth dose is administered, so that total and unit costs may be slightly underestimated. Nevertheless, maternities only provide two out of 22 doses in the immunization schedule and are larger in size compared with the facilities in our sample, so that maternities would be expected to deliver immunization at a lower cost. Consequently, our underestimations are expected to be small. Secondly, our definition of the FIC is not fully compatible with the national definition used in Moldova. Consequently, careful interpretation is needed when comparing with the findings from other national as well as internationally available data. Finally, labor cost estimates could be affected by recall bias related to a time spent on immunization activities, although efforts were made to minimize this bias by organizing group discussions at the facility to reach consensus among staff.

\section{Conclusions}

Our findings could inform policy discussions within Moldova around increasing immunization coverage rates while running the program more efficiently and sustainably. At the same time increasing coverage rates through sustainable 
financing is a global concern, well beyond Moldova. Our study noted variations in total immunization costs and unit costs at the facility level and variation in the facility performance measured by DTP3 coverage rate. The analysis allows hypothesizing that characteristic of a facility may have influenced achieved DPT3 coverage rates. However available evidence also indicates that immunization coverage rates are as well dependent on socioeconomic characteristics of the population [21-23] on their proximity to health facility $[24,25]$ etc. Therefore, it would be valuable to evaluate other context specific determinants (e.g. population and geography characteristics) in a multivariate model to establish causal links between the factors determined by our bi-variate analysis for the facility performance and costs. Such research could provide an additional valuable contribution to the Global evidence in addition to this study.

\section{Acknowledgements}

This study would have not been possible without the continuous support of the Ministry of Health of the Republic of Moldova, EPI manager, directors of District Public Health Centers and health care providers who gave their time as well as allowed access to facilities and facility records. Therefore, we would like to acknowledge their invaluable support in carrying out this work. We would like to thank Bill \& Melinda Gates Foundation for providing financial support. And in particular, we would like to acknowledge the support of Logan Brenzel and Damian Walker, steering committee members and other country teams that commented on an earlier draft of this paper and provided valuable inputs and guidance to the study team. 


\section{Conflict of interest}

The authors have declared that they have no conflict of interest. [1] The World bank. World Development Report 1993 : Investing in Health. New York : World Bank and Oxford University Press, 1993.

[2] Brenzel L, Wolfson LJ, Fox- Rushby J, Miller M, Halsey N. Vaccine Preventable Diseases. [book auth.] Breman JG, Measham AR, Alleyne G, Claeson M, Evans DB, Jha P, Mills A, Musgrove P. Jamison DT. Disease Control Priorities in Developing Countries. 2nd edition. Washington DC: World Bank 2006, Chapter 20, p. 389-411. [3] Stephen S Lim PhD, David B Stein BA, Alexandra Charrow BA, Prof Christopher JL Murray MD. Tracking progress towards universal childhood immunisation and the impact of global initiatives: a systematic analysis of three-dose diphtheria, tetanus, and pertussis immunisation coverage. The Lancet - 13 December 2008 ( Vol. 372, Issue 9655, Pages 2031-2046 )

[4] Government of Republic of Moldova. GAVI Alliance. Comprehensive Multi-Year Plan for Immunization Program 2011-2015 [Online] [Cited: April 04, 2013.]

[5] www.transmonee.org (Last accessed on August 5, 2014)

[6] Brenzel L, Young D, Walker DJ. Costs and Financing of Routine Immunization: Approach and Selected Findings of a Multi- Country Study (EPIC). Vaccine 2015. In press.

[7] BMGF; Common approach for the costing and financing analyses of routine immunization and new vaccine introduction costs; Unpublished paper; 2013.

[8] WHO Organization Health World, IVB Immunization Vaccines \& Biologicals. World Health Organization.[Online]December 2006.[Cited:November 14,2013.]

[9] Hoang MV, Nguyen TBY, Kim BG, Dao LH, Nguyenc TH, Wright P. Cost of Providing the Expanded Programme on Immunization: Findings from a Facility- 
Based Study in Viet Nam, 2005. Bulletin of the World Health Organization 2008; 86(6):429-434.

[10] Drummond MF, Sculpher MJ, Torrance GW, O Brien MJ, Stoddart GL. Methods for the Economic Evaluation of Health Care Programmes. Second edition. New York : Oxford University Press 2005.

[11] Walker D, Kumaranayake L. Allowing for Differential Timing in Cost Analyses: Discounting and Annualization. London UK: Oxford University Press, Health Policy and Planning 2002; 17(1):112-118.

[12] Gotsadze G., Goguadze K., Chikovani I., Maceira D. Analyses of the Costs and Financing of Routine Immunization Program and New vaccine Introduction in Moldova Technical Report. Curatio International Foundation 2014.

[13] WHO, World Health Organization. Product Information Sheets (PIS) [Online] [Cited:April12,2013.]

http:// www.who.int/immunization_standards/vaccine_quality/ pis/ en/ .

[14] Robert L. Robertson, Joe H. Davis., Kebba Jobe; Service volume and other factors affecting the cost of immunizations in the Gambia. Bulletin of the World Health Organization 1984; 62 (5):729-736.

[15] Walker D, Mosqueira NR, Penny ME, Lanata CF, Clark AD, Sanderson CFB, FoxRushby JA. Variation in the costs of delivering routine immunization services in Peru. Bulletin of the World Health Organization 2004; 82:676-682.

[16] Khan, M., S. Khan, D. Walker, J. Fox-Rushby, F. Cutts, and S.M. Akramuzzaman; Cost of Delivering Child Immunization Services in Urban Bangladesh: A Study Based on Facility-level Surveys. J HEALTH POPULNUTR, Dec 2004; 22(4):404-412.

[17] WHO. Task Shifting. Global recommendations and guidelines. 2008 
[18] Bishai D, McQuestion M, Chaudhry R, Wigton A. The Costs of Scaling Up Vaccination in the World's Poorest Countries 2006; Health Affairs; 25(2):404-412.

[19] WHO, GAVI. Moldova Graduating from GAVI support: Situation Analysis and Transition Plan. 2012.

[20] WHO, UNICEF. [Online] Immunization Summary - A Statistical Reference Containing Data Through 2011. [Cited: October 08, 2013.]

[21] Mitchell S, Andersson N, Ansari NM et al. Equity and vaccine uptake: a crosssectional study of measles vaccination in Lasbela District, Pakistan. BMC International Health and Human Rights 2009; 9(Suppl.1):S1-S7

[22] IA, Semali. Trends in Immunization Completion and Disparities in the Context of Health Reforms: The case study of Tanzania. BMC Health Services Research 2010; 10:299.

[23] Tarlier DS, Browne AJ, Johnson J. The Influence of Geographical and Social Distance on Nursing Practice and Continuity of Care in a Remote First Nations Community. Canadian Journal of Nursing Research 2007; 39(3):126-148.

[24] Kanta J, Bhuiya A, Streatfield K, Chakrabarty N. The Immunization Programme in Bangladesh: Impressive Gains in Coverage, but Gaps Remain. Health Policy and Planning 1999; 14(1):49-58.

[25] Bhuiya A, Bhuiya I, Chowdhury M. Factors Affecting Acceptance of Immunization Among Children in Rural Bangladesh. Health Policy and Planning 1995; 10(3):304-312. 
Table 1 Cost structure by Facility Type and Facility Scale

\begin{tabular}{|c|c|c|c|c|c|c|c|c|}
\hline \multirow[b]{2}{*}{ Item } & \multicolumn{4}{|c|}{ Facility Type } & \multicolumn{4}{|c|}{ Facility Scale } \\
\hline & FMC & HC & OFD & HO & Low & Medium & High & Total \\
\hline Recurrent cost (\%) of Total & 89.7 & 88.7 & 85.4 & 77.2 & 76.9 & 87.7 & 90.7 & 83.7 \\
\hline Salaried Labor & 70.4 & 73.2 & 68.1 & 54.4 & 55.5 & 71.4 & 73.3 & 65.1 \\
\hline $\begin{array}{l}\text { Vaccines and injection } \\
\text { supplies }\end{array}$ & 14.4 & 8.2 & 8.8 & 8.3 & 8.1 & 8.6 & 10.6 & 8.7 \\
\hline Utilities and communications & 2.7 & 4.8 & 5.9 & 8.5 & 8.3 & 5.1 & 5.0 & 6.4 \\
\hline Printing & 1.1 & 0.5 & 0.1 & 0.0 & 2.4 & 1.5 & 0.5 & 1.7 \\
\hline Cold chain energy & 0.1 & 0.5 & 0.5 & 1.8 & 1.4 & 0.5 & 0.2 & 0.8 \\
\hline Other recurrent & 1.0 & 1.5 & 2.0 & 4.2 & 1.2 & 0.6 & 1.1 & 1.0 \\
\hline Capital cost (\%) of Total & 10.3 & 11.3 & 14.6 & 22.8 & 23.1 & 12.3 & 9.3 & 16.3 \\
\hline Building & 7.1 & 5.8 & 9.2 & 15.3 & 15.2 & 7.5 & 5.7 & 10.3 \\
\hline Cold chain equipment & 0.2 & 0.8 & 2.3 & 4.6 & 4.5 & 1.6 & 0.6 & 2.7 \\
\hline Other capital costs & 3.0 & 4.7 & 3.1 & 2.9 & 3.4 & 3.2 & 3.0 & 3.3 \\
\hline TOTAL \% & 100 & 100 & 100 & 100 & 100 & 100 & 100 & 100 \\
\hline Unit Cost per Dose US\$ & 10.4 & 19.4 & 18.5 & 18.7 & 20.5 & 18.3 & 14.5 & 18.4 \\
\hline
\end{tabular}


Table 2: Facility Unit Costs by Type of facility, Location and Facility Scale

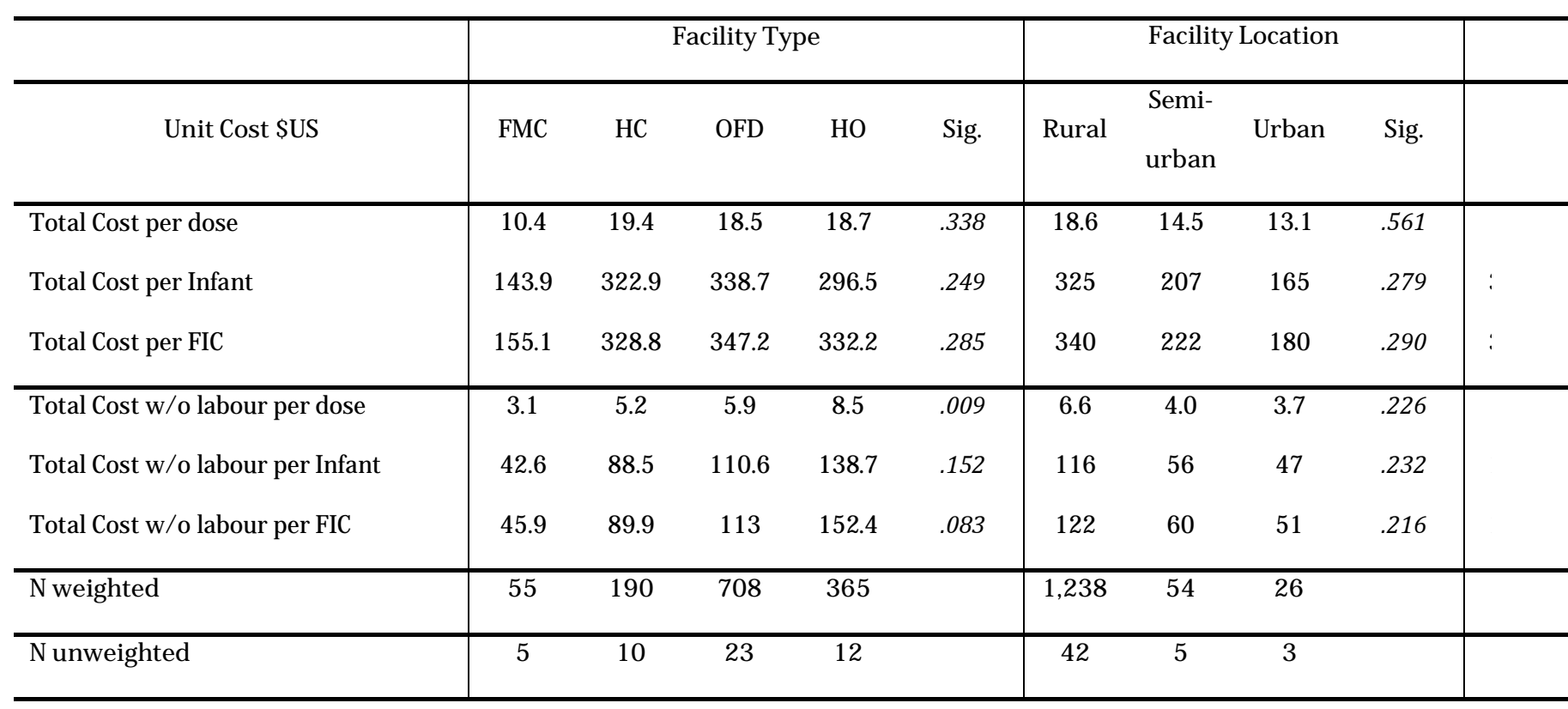


Figure 1: Total costs by type of facility and DTP3 coverage $(\%)^{6}$

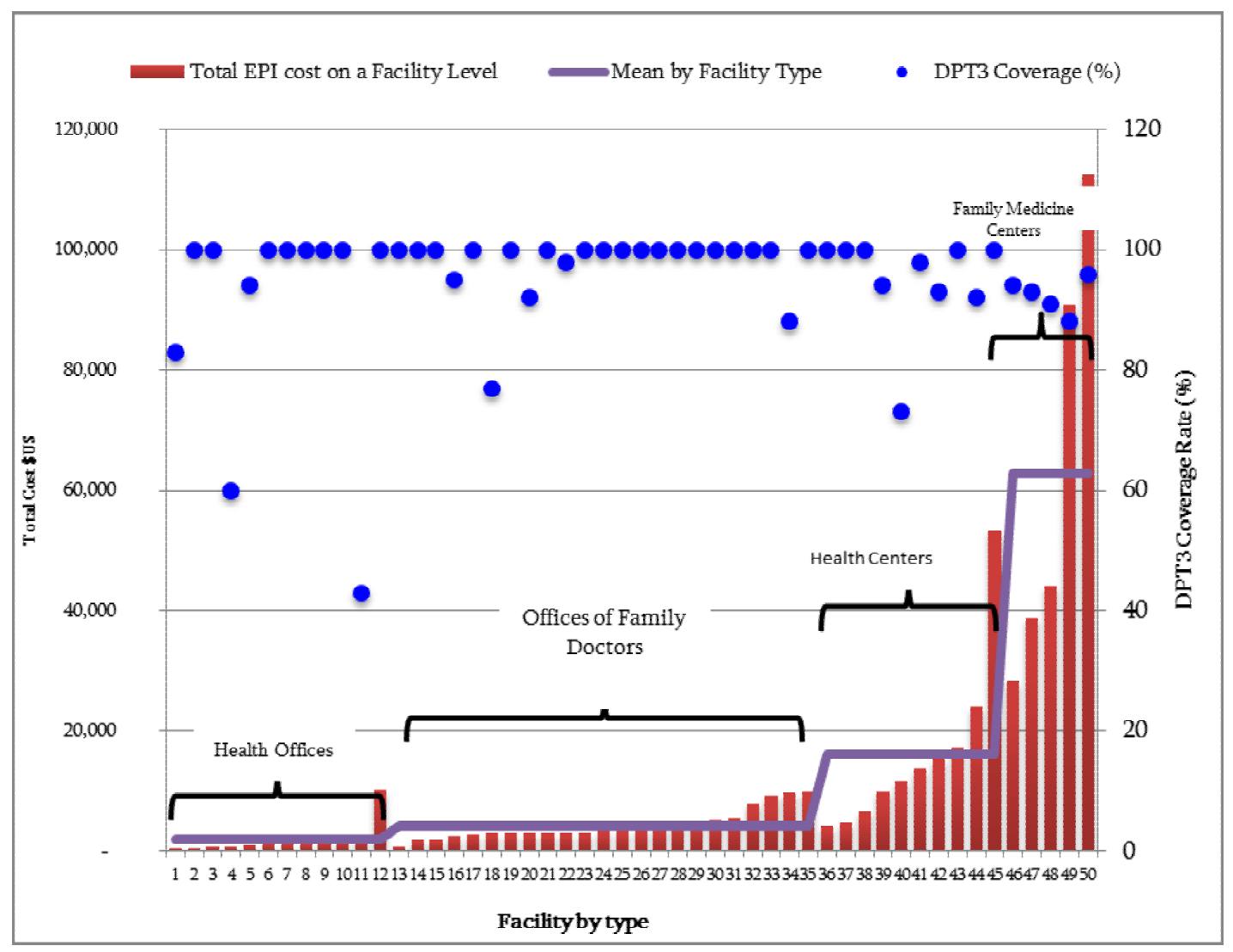

${ }^{6}$ The fact that the denominators of target population differ by facility type the low coverage rates at smaller facilities mean fewer individuals compared to bigger facilities. 
Figure 2: Total cost without labour per Dose by facility scale

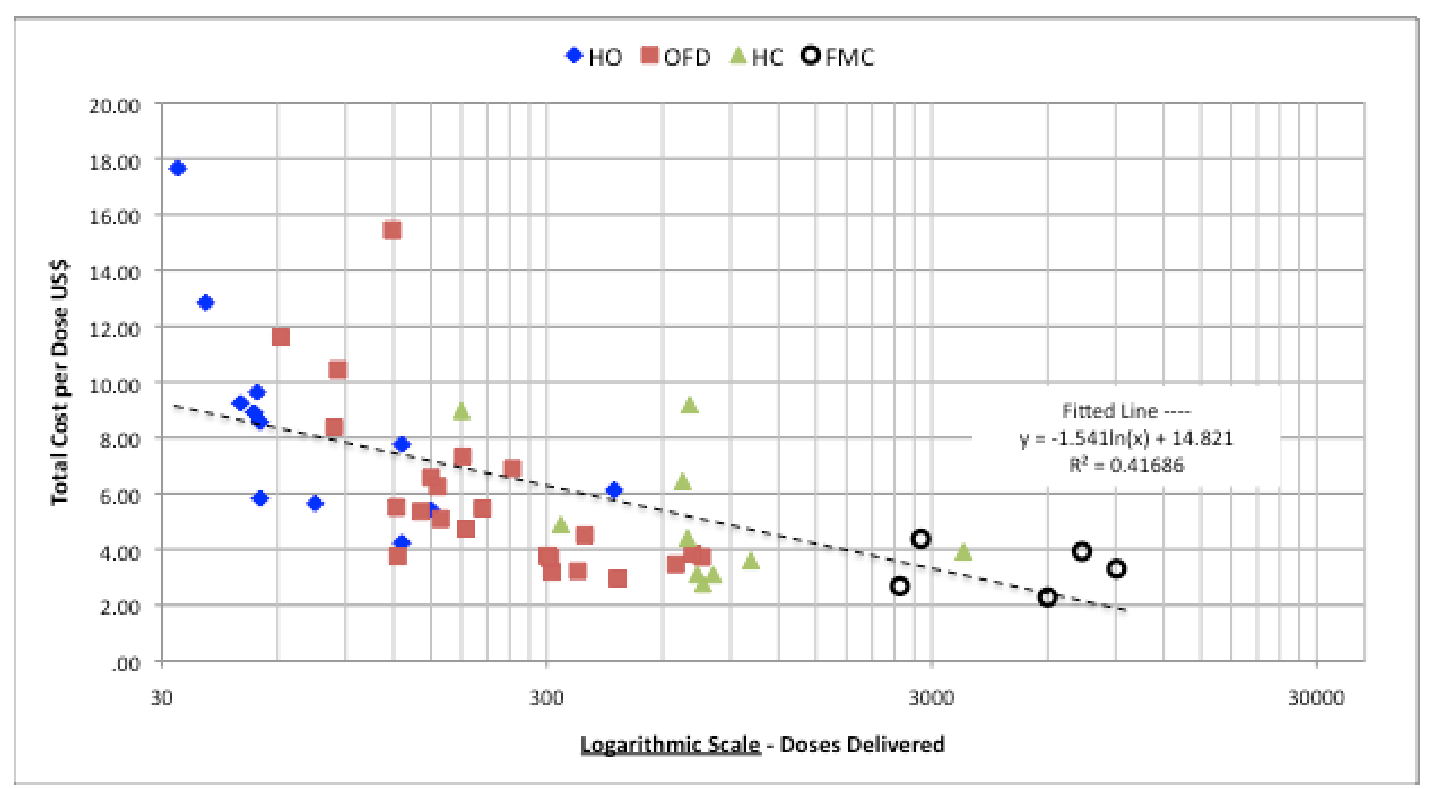


2 Figure 3 Total Cost per Dose and DTP3 coverage rate by type of facility

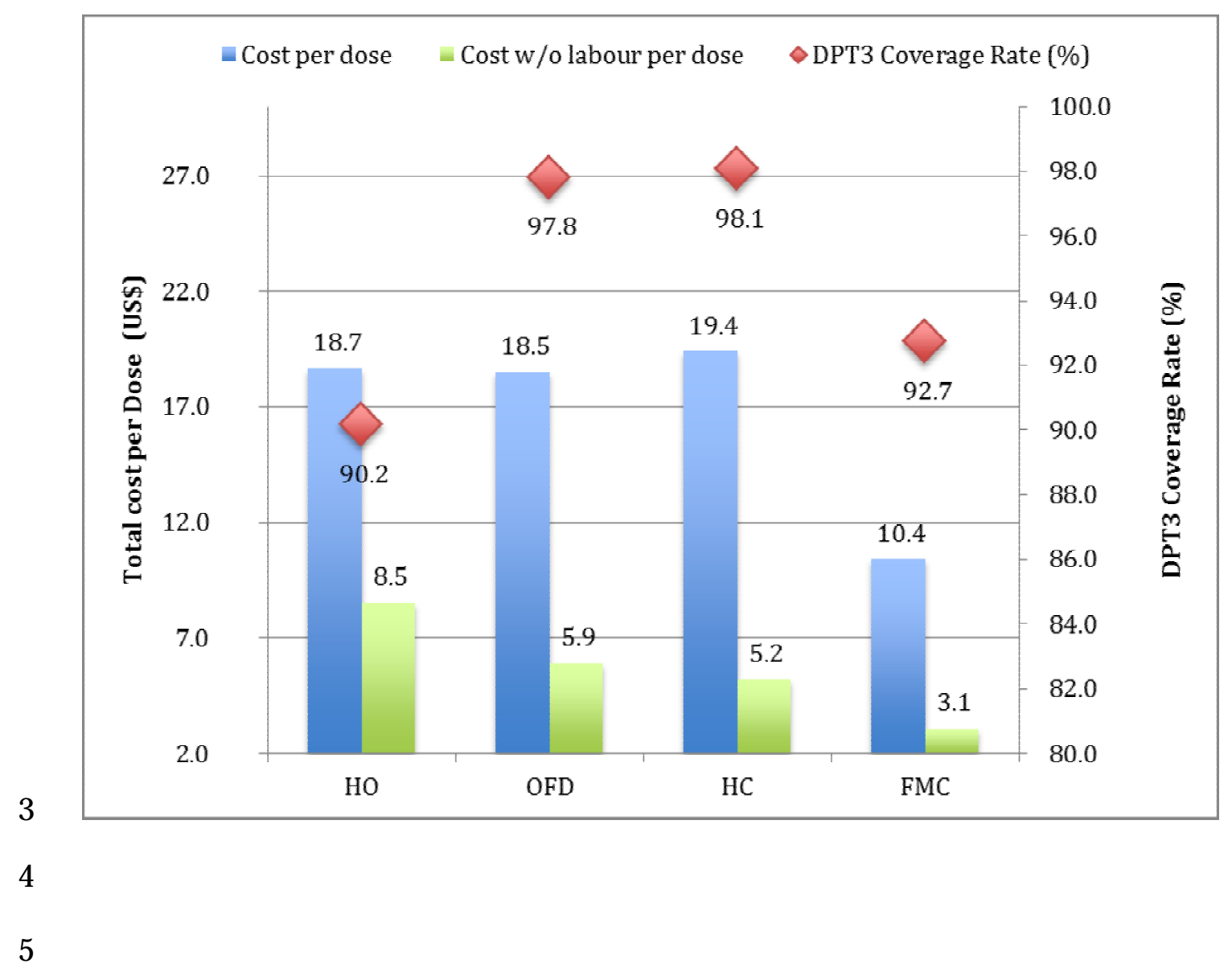

